\title{
Sustainable source of omega-3 eicosapentaenoic acid from metabolically engineered Yarrowia lipolytica: from fundamental research to commercial production
}

\author{
Dongming Xie • Ethel N. Jackson • Quinn Zhu
}

Received: 29 October 2014 / Revised: 4 December 2014 / Accepted: 9 December 2014 / Published online: 8 January 2015

(C) The Author(s) 2015. This article is published with open access at Springerlink.com

\begin{abstract}
The omega- 3 fatty acids, cis-5, 8, 11, 14, and 17eicosapentaenoic acid (C20:5; EPA) and cis-4, 7, 10, 13, 16, and 19-docosahexaenoic acid (C22:6; DHA), have wideranging benefits in improving heart health, immune function, mental health, and infant cognitive development. Currently, the major source for EPA and DHA is from fish oil, and a minor source of DHA is from microalgae. With the increased demand for EPA and DHA, DuPont has developed a clean and sustainable source of the omega-3 fatty acid EPA through fermentation using metabolically engineered strains of Yarrowia lipolytica. In this mini-review, we will focus on DuPont's technology for EPA production. Specifically, EPA biosynthetic and supporting pathways have been introduced into the oleaginous yeast to synthesize and accumulate EPA under fermentation conditions. This Yarrowia platform can also produce tailored omega-3 (EPA, DHA) and/or omega-6 (ARA, GLA) fatty acid mixtures in the cellular lipid profiles. Fundamental research such as metabolic engineering for strain construction, high-throughput screening for strain selection, fermentation process development, and process scale-up were all needed to achieve the high levels of EPA titer, rate, and yield required for commercial application. Here, we summarize how we have combined the fundamental bioscience and the industrial engineering skills to achieve large-scale production of Yarrowia biomass containing high amounts of EPA, which led to two commercial products, New Harvest ${ }^{\mathrm{TM}}$ EPA oil and Verlasso ${ }^{\circledR}$ salmon.
\end{abstract}

Keywords Yarrowia lipolytica . Omega-3 fatty acid . Metabolic engineering · Fermentation - Commercialization

D. Xie $\cdot$ E. N. Jackson $\cdot$ Q. Zhu $(\bowtie)$

Biotechnology, Central Research and Development, E.I. du Pont de Nemours and Company, Wilmington, DE, USA

e-mail: quinn.zhu@dupont.com

\section{Introduction}

Omega-3 fatty acids refer to the long-chain polyunsaturated fatty acids (LCPUFA) with the first $\mathrm{C}=\mathrm{C}$ double bond at the n-3 position, i.e., the third carbon from the methyl end of the carbon chain. There have been many clinical studies showing a wide range of health benefits from the omega-3 LCPUFAs, especially the eicosapentaenoic acid (C20:5; EPA) and docosahexaenoic acid (C22:6; DHA) (Martins et al. 2013; Chacon-Lee and Gonzalez-Marino 2010; Kapoor and Patil 2011; Calder 2006). In general, it is believed that EPA is able to improve cardiovascular health, mental health, and immune function, while DHA is able to improve mental health and infant cognitive development. The Japan EPA Lipid Intervention Study (JELIS) showed that EPA is a promising treatment for prevention of major coronary events (Yokoyama et al. 2007). The AMR101 study also showed that pure EPA fatty acid significantly reduced triglyceride levels in adult patients with severe hypertriglyceridemia (Ballantyne et al. 2012). The human body can only inefficiently synthesize the EPA and DHA from omega-3 alpha-linolenic acid (C18:3; ALA) but cannot de novo synthesize them (Kapoor and Patil 2011). EPA and DHA in our bodies are largely from our foods, especially cold-water oceanic fishes (Martins et al. 2013).

EPA and DHA are synthesized de novo in marine microorganisms and phytoplankton. Some ocean fishes (e.g., wild salmon, Pacific sardine) can accumulate significant amounts of EPA and DHA by eating microalgae cells in the ocean. Fish oil is the main source of EPA and DHA; however, its availability and sustainability have been questioned due to overfishing and contamination in the ocean environment. To overcome this limitation, biotechnology industries started to produce DHA directly from microalgae in large-scale fermentation process (Kyle 2001). However, there is no large scale land-based EPA production from wild-type organisms, because EPA productivity is too low to meet commercial targets. 
Consequently, DuPont initiated a research program to develop a sustainable EPA source by metabolic engineering of Yarrowia lipolytica (Fig. 1). We now have successfully demonstrated that the engineered $Y$. lipolytica strains can produce various omega-3 and omega- 6 fatty acids. Our first targeted product for commercialization was EPA due to its unique health benefits and the lack of a land-based sustainable supply.

This mini-review summarizes DuPont's results in both metabolic engineering research and fermentation process development for commercial production of EPA. The engineered Gen I strain Y4305 (Xue et al. 2013a) produced EPA at more than $15 \%$ of its dry cell weight (DCW); the Gen II strain Z1978 (Hong et al. 2011a) produced EPA at more than $20 \%$ of its DCW, the Gen III HP strain Z5567 (Hong et al. 2011a) produced EPA at more than $25 \%$ of its DCW. The purified lipids from EPA producing strains have been used to develop a commercial product, New Harvest ${ }^{\mathrm{TM}}$ EPA oil, for a human nutritional supplement. The high-EPA biomass of these strains has also been used to raise Verlasso ${ }^{\circledR}$, a sustainably farmed salmon. This is a good example of a yeast metabolically engineered to produce a commercial product to replace a fish-derived product. Our work has paved the way for further improvement of EPA production strains and development of strains with desired fatty acid compositions for specific applications. This advanced Yarrowia biotechnology platform can also be used for production of other high-value products.

\section{Y. lipolytica is a safe and productive host for EPA production}

Appropriate host selection is a pre-request for the success of this project. The number one selection guideline is the safety of the organism. Y. lipolytica is found primarily in foods with high proportions of fat and/or protein, particularly in (fermented) dairy products and meat. Extensive research and analyses demonstrated that $Y$. lipolytica is a safe organism to be used for industrial applications (Groenewald et al. 2014). $Y$. lipolytica was first used to produce single cell protein using cheap and abundant $n$-paraffins as the sole carbon source for animal feeds under the trade name of Toprina (Ratledge 2005); it was also classified as "Generally Recognized as Safe (GRAS)" for commercial production of food grade citric acid (US Food and Drug Administration list of microbialderived ingredients approved for use in food; Title 21, Part 173, Sec. 165). Other applications include production of erythritol, wax esters, 2-ketoglutaric, 2-hydroxyglutaric, and isopropylmalic acids and secretion of heterologous proteins, including several food enzymes (Ratledge 2005; Groenewald et al. 2014).

$Y$. lipolytica has an established history of robust fermentation performance. The cell density can reach more than $100 \mathrm{~g}$ DCW/L with carbohydrates such as glucose, fructose, glycerol, or fatty acids as sole carbon source. Most Y. lipolytica strains are haploid (Barth and Gaillardin 1997), but can also exist in diploid form. Depending on growth conditions, $Y$. lipolytica cells can differentiate into yeast, pseudomycelium, and true mycelial forms (Pérez-Campo and Domínguez 2001; Szabo and Stofaníková 2002). $Y$. lipolytica has a metabolism that is well suited to fatty acid production and lipid accumulation (Blank et al. 2005; Nicaud 2012; Tai and Stephanopoulos 2013); it has also been used as host organism for sustainable production of biodiesel, functional dietary lipid compounds, and other value-added compounds (Beopoulos et al. 2009 and 2010; Abghari and Chen 2014).

Some $Y$. lipolytica strains are oleaginous organisms that can accumulate up to more than $30 \%$ DCW as storage triglycerides (TAG) under the condition of nitrogen starvation and glucose excess. Although the central carbon metabolism of $Y$. lipolytica is similar to other yeasts, it has significant regulatory differences. It also has high flux for the pentose phosphate pathway that generates cofactor NADPH to support lipid biosynthesis (Blank et al. 2005). The lipid from glucose-grown cells is comprised mainly of TAG in which oleic acid (C18:1 n-9) and linoleic acid (LA, C18:2 n-6) are the two major fatty acids (Xue et al. 2013a).

There are six chromosomes in Y. lipolytica. A complete genome sequence of strain CLIB122 has been published (Dujon et al. 2004). It has a total of about $20 \mathrm{Mb}$ DNA that encodes about 6,500 genes. There is no extra-chromosomal plasmid discovered in wild-type strains. Genetic transformation occurs when exogenous DNA integrates into the genome by homologous and nonhomologous recombination. $Y$. lipolytica has been used as a model system for studying hydrophobic substrate utilization, peroxisome biogenesis, lipid metabolism, and bio-lipid production (Nicaud 2012; Tai
Fig. 1 Fermentation production of eicosapentaenoic acid (EPA, C20:5 n-3) from sugar by metabolically engineered Yarrowia lipolytica strains

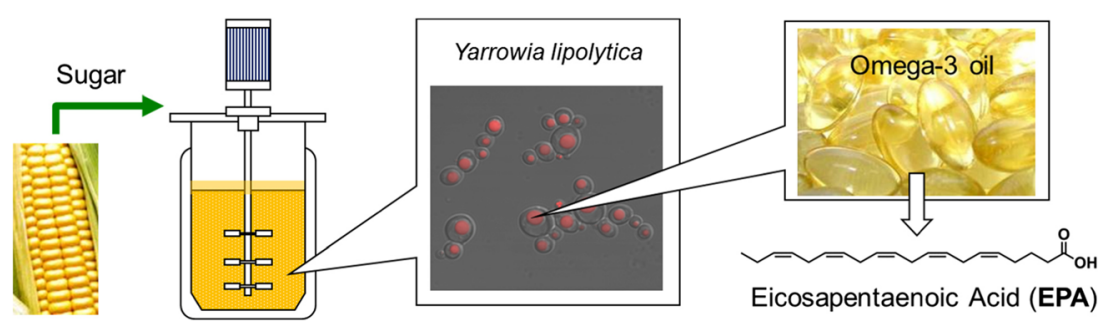


and Stephanopoulos 2013). It is easy to develop auxotrophic mutants for $Y$. lipolytica. Transformants can be selected by complementation of auxotrophic mutations, and the use of antibiotic resistance genes as selectable markers is not required. The auxotrophic markers most commonly used are the LYS5 gene coding for saccharopine dehydrogenase (Xuan et al. 1988), the LEU2 gene coding for beta-isopropylmalate dehydrogenase (Davidow et al. 1987a, b), and the URA3 gene encoding for orotidine 5'-monophosphate decarboxylase (Mauersberger et al. 2001). The counter selection system of the URA3 gene and 5-fluoroorotic acid (5-FOA) allows multiple rounds of integration of functional genes into the $Y$. lipolytica genome thereby to introduce many copies of foreign genes (Barth and Gaillardin 1996; Zhu et al. 2010). In the last 30 years, $Y$. lipolytica is one of the most studied unconventional yeasts (Nicaud 2012). There is extensive knowledge accumulated on its genetics, molecular biology, and physiology. Most of these studies suggest that $Y$. lipolytica is a good model system not only for basic scientific research but also for industrial applications.

We collected and screened over 40 different $Y$. lipolytica strains from various public depositories all over the world for their fermentation performances and ability to accumulate omega-3 fatty acids such as EPA and DHA when these fatty acids are fed as substrates. After a careful execution of statistically designed experiments, the strain American Type Culture Collection (ATCC) \#20362 achieved our fermentation performance targets: DCW greater than $100 \mathrm{~g} / \mathrm{L}$, lipid content greater than $30 \% \mathrm{DCW}$, and lipid productivity greater than $1 \mathrm{~g} / \mathrm{L} / \mathrm{h}$. We then selected ATCC \#20362 strain for pathway engineering. The genome sequence of strain ATCC \#20362 has more than $99 \%$ identity with the genome sequence of strain CLIB122 (www.genolevures.org/yali.html). Like French strain W29 and German strain H222, strain ATCC \#20362 does not contain retrotransposon-like element (Ylt1) that exists in strain CLIB122 and some American strains (Schmid-Berger et al. 1994; Mauersberger et al. 2001).

\section{EPA biosynthetic pathways}

Wild-type $Y$. lipolytica does not make any omega-3 fatty acids. The fatty acid profile of the wild-type strain ATCC \#20362 (Zhu et al. 2010; Xue et al. 2013a) shows that it can synthesize linoleic acid (LA, C18:2 n-6). There are different published biosynthetic routes to make EPA, the anaerobic polyketide synthase pathway (Metz et al. 2001) or an aerobic desaturase and elongase pathway (Meesapyodsuk and Qiu 2012). The microalgae Crypthecodinium cohnii and Schizochytrium sp. used for DHA commercial production use the polyketide synthase pathway. Many microalgae and some marine bacteria also use the polyketide synthase pathway to synthesize EPA (Metz et al. 2001; Wen and Chen 2005). However, the rate, titer, and yield from these organisms could not meet the requirement for commercial production. The aerobic pathway (Fig. 2) can be further classified into a $\Delta 6$-desaturase pathway (the $\Delta 6$ pathway found in algae, mosses, fungi, and others) or a $\Delta 9$-elongase and $\Delta 8$-desaturase (Wallis and Browse 1999) pathways (the $\Delta 9$ pathway). The $\Delta 9$ pathway (Sayanova and Napier 2004) has been found in some species from Prymnesiophyceae (Pavlova, Isochrysis), Acanthamoebae (e.g., Acanthamoeba) and Euglenophyceae (e.g., Euglena).

The differences between the $\Delta 6$ and $\Delta 9$ pathways are the first two steps. In the $\Delta 6$ pathway, the first step is the $\Delta 6$ desaturase to covert the LA and/or ALA to gamma-linolenic acid (GLA, 18:3 n-6) and/or stearidonic acid (STA, 18:4 n-3). The second step is the $\mathrm{C}_{18 / 20}$ elongase to convert the GLA and/or STA to dihomo-gamma-linoleic acid (DGLA, 20:3 n6) and/or eicosatetraenoic acid (ETA, 20:4 n-3). In the $\Delta 9$ pathway, the first step is the $\Delta 9$ elongase to convert LA and/or ALA to eicosadienoic acid (EDA, 20:2 n-6) and/or eicosatrienoic acid (ETrA: 20:3 n-3). The second step is the $\triangle 8$ desaturase to convert EDA and/or EtrA to DGLA and/or ETA. The last two steps are the same between these two pathways.

It should be noted that the $\Delta 15$ desaturase and $\Delta 17$ desaturase are omega-3 desaturases; these two enzymes convert the omega- 6 fatty acids into omega-3 fatty acids. The $\Delta 15$ desaturase converts LA to ALA. So far, there is no $\Delta 15$ desaturase found to convert LA to ALA with $100 \%$ efficiency, so transformed cells with a heterologous $\Delta 15$ desaturase gene will contain both LA and ALA. Therefore, the $\Delta 6$ and $\Delta 9$ pathways can simultaneously use both LA and ALA as primary substrates. Apart from its primary function to convert ARA to EPA, most $\Delta 17$ desaturases can also convert EDA to ETrA with less efficiency.

All these desaturation and elongation enzymes carry out their reactions in the endoplasmic reticulum (ER) membrane (Meesapyodsuk and Qiu 2012). It is believed that the substrates of desaturases and elongases are in the form of phospholipid and acyl-CoAs, respectively. Elongation usually is the rate-limiting step of the aerobic pathways for EPA biosynthesis. Nevertheless, introduction of either the $\Delta 6$ or $\Delta 9$ pathway genes into the wild-type strain should allow the production of EPA through desaturation and elongation of the native fatty acid species.

\section{Toolbox for metabolic engineering of $Y$. lipolytica for EPA production}

Before we started our project, the $Y$. lipolytica transformation system (Chen et al. 1997) had been established, and a lowcopy replication plasmid had been developed (Fournier et al. 1993). Scientists were trying to use it as a host for efficient secretion of expressed heterologous proteins (Davidow et al. 
Fig. 2 Metabolic engineering in Yarrowia lipolytica for omega-3 EPA and DHA production. The native fatty acid pathway is indicated in gray and the engineered pathway for omega-3 EPA and DHA production is indicated in green (color figure online)

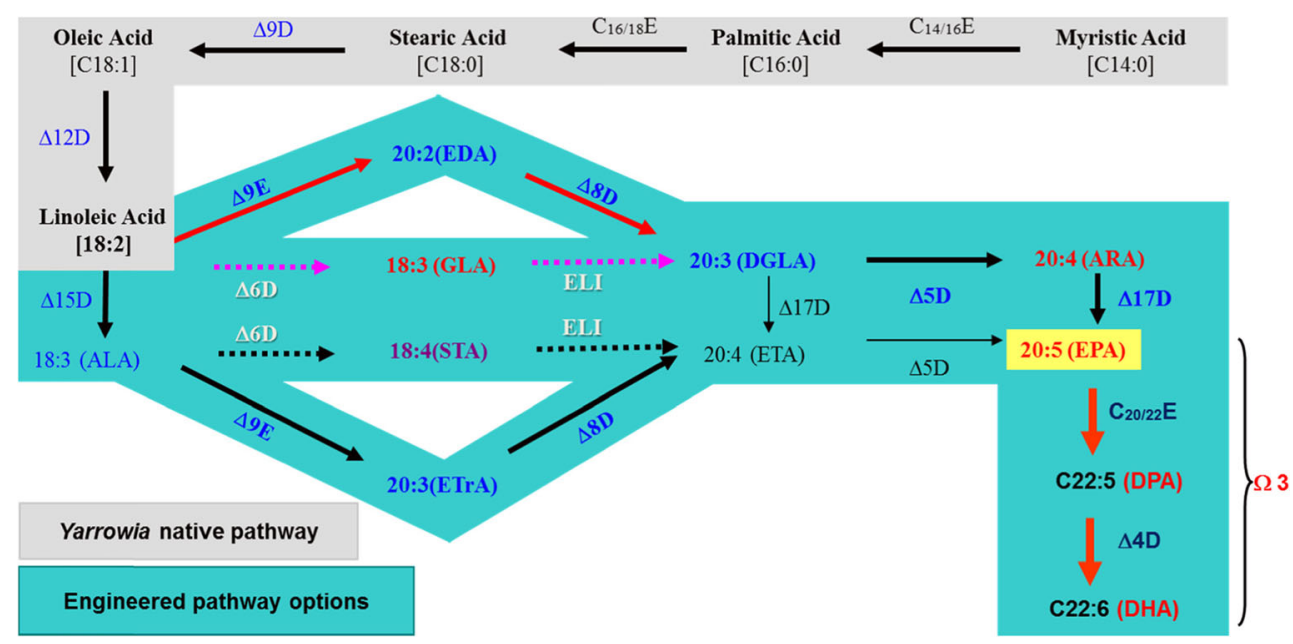

1987a, b) or as a system to study certain biological functions. There were limited genetic elements such as promoters and terminators available that are necessary for metabolic engineering (Muller et al. 1998; Juretzek et al. 2001). We quickly found that the expression of an introduced gene was higher when it integrated into the genome than when it was in the low-copy replication plasmid. In order to develop Y. lipolytica as a host for EPA production by metabolic engineering, we pursued several research goals simultaneously.

1. Enrich the tool box for metabolic engineering

2. Development and test strategies for introducing multiple copies of foreign genes into Y. lipolytica

3. Establish a screening system for strain development

4. Develop the fermentation process

To demonstrate that $Y$. lipolytica can be engineered to produce EPA, we licensed the promoter of the translation elongation factor (TEF) gene of Y. lipolytica from Novozyme Corporation (Muller et al. 1998) and used it to drive the expression of the individual genes encoding $\Delta 6$ desaturase, $\mathrm{C}_{18 / 20}$ elongase, $\Delta 5$ desaturase, and $\Delta 17$ desaturase that we licensed from Ross Division of Abbott Laboratories (Knutzon et al. 1998; Picataggio et al. 2007). Integration of a single copy of these four gene expression cassettes into the genome of $Y$. lipolytica strain ATCC \#20362 resulted in the synthesis of EPA at about $3 \%$ of the total fatty acid methyl esters (FAME), with $34 \%$ of all fatty acids derived from the engineered pathway, and the majority was GLA (Zhu et al. 2010). This result demonstrated that $Y$. lipolytica could be engineered to produce EPA and suggested that additional engineering improvements were needed to (1) increase the carbon flux into the engineered pathway, (2) improve the efficiency of the $\mathrm{C}_{18 / 20}$ elongases to convert more GLA into DGLA, and (3) enhance the expression of other pathway genes.
To enhance the expression of foreign genes in Y. lipolytica, we isolated a set of promoters that are at least as strong as the TEF promoter (Muller et al. 1998). The promoters we isolated are from the genes encoding export protein (EXP, YALI0C12034g), fructose 1,6-bisphosphate aldolase (FBA1; YALI0E26004g), glycerol-3-phosphate- $O$-acyltransferase (GPAT, YALI0C00187g), phosphoglycerate mutase (GPM1; YALI0B02728g), glycerol-3-phosphate dehydrogenase (GPD1; YALI0B02948g), and an ammonium transporter (YAT1, YALI0E27181g). All these promoter activities were compared with TEF promoter by quantitative fluorometric assays of the beta-glucuronidase (GUS) reporter (Jefferson et al. 1987) driven by each individual promoter. The results showed that the FBA1 promoter was the strongest among the six promoters. The GPMI promoter was as strong as the TEF promoter, the GPD1 promoter was 2.5 times stronger than the GPM1 promoter, and the $F B A 1$ promoter activity was 5.5 and 2.2 times stronger than the GPDI and GPM1 promoters, respectively.

In the N-terminal coding region of the $F B A 1$ gene, there is a 102 base pair intron located between the codons for amino acids 20 and 21. Fusion of the FBA1 promoter plus the $N$ terminal coding region covering the first 23 amino acids and the intron $\left(F B A 1_{\text {in }}\right)$ with the GUS reporter gene resulted in GUS activity about five times greater than the FBA promoter alone. As in the case of the FBA1 gene, the GPD1 gene also has an intron located in its N-terminus, which can significantly enhance the GPD promoter activity (Picataggio and Zhu 2007). The $N$-terminal coding regions with introns of $F B A 1$ and GPD1 genes enhanced the activity of GPM promoter when chimeric promoters were constructed (Hong et al. 2011b).

The YAT1 promoter has a unique feature, since it has almost no activity under normal growth conditions, but under nitrogen-limiting conditions, its activity increased approximately 35-fold (Xue and Zhu 2012). This promoter is useful 
for directing the expression of omega- 3 biosynthetic genes, because lipid synthesis and accumulation need nitrogen starvation. The relative strength of these promoters in nitrogenlimiting conditions was determined by quantitative GUS assays, and they are as follows: $\mathrm{FBA}_{\text {in }}>\mathrm{YAT} 1>\mathrm{FBA}>\mathrm{GPD}$, $\mathrm{EXP}>\mathrm{GPAT}>\mathrm{GPM}=\mathrm{TEF}$.

There are about 6,500 genes in Y. lipolytica. The promoter of each gene has a unique property. A diverse promoter library could also be generated through random mutagenesis using a specific promoter as template (Alper et al. 2005). Promoter characteristic studies are required before they can be utilized to maximize the expression of targeted genes in desired conditions.

To improve the expression of foreign genes in Y. lipolytica, we developed a program to codon-optimize all the genes according to the codon usage pattern and GC content of highly expressed genes of $Y$. lipolytica. There are two $\Delta 6$-desaturase genes from Mortierella alpina and Saprolegnia diclina, two $\mathrm{C}_{18 / 20}$-elongase genes from M. alpina and Thraustochytrium aureum, three $\Delta 5$-desaturase genes from Isochrysis galbana, M. alpina, and $S$. diclina, and one $\Delta 17$-desaturase gene from S. diclina (Zhu et al. 2010, Knutzon et al. 1998, Picataggio et al. 2007). The optimized genes also contain the consensus sequence (5'-ACCATGG-3') around the "ATG" translation initiation codon. We discovered that the substrate conversion was increased in almost all of the codon-optimized genes except the $\Delta 5$-desaturase gene derived from $M$. alpina. The improved substrate conversion efficiency of these "codonoptimized" genes is hypothesized to result from more efficient translation of their encoded mRNAs in Y. lipolytica. Right now, the genes introduced into Y. lipolytica for strain construction are all codon-optimized, and each of the synthetic genes were designed with an $\mathrm{NcoI}$ site around its translation initiation site and a NotI site after its stop codon. The modular structure of these coding regions, promoters, and terminators is easy to swap for construction of expression constructs with different configurations (Fig. 3).

From our early experiments, it was clear that we needed to introduce multiple copies of genes involved in EPA biosynthetic pathway for production of high amounts of EPA in engineered $Y$. lipolytica cells. Using the URA3 gene and its counter selection by 5 -fluoroorotic acid (FOA), we developed a system that can integrate many copies of foreign genes into the genome of $Y$. lipolytica via sequential integrations. FOA is toxic to yeast cells that possess a functional URA3 gene, and this compound is not toxic to the yeast cells with an inactivated ura3 gene (Barth and Gaillardin 1996; Zhu et al. 2010). As shown in Fig. 4, the native URA3 gene can be knocked out by using a DNA fragment with mutated ura3, and the transformants growing on FOA plates will have the ura3-phenotype. A cluster of multiple chimeric genes (or a single chimeric gene) and a new URA3 gene can be integrated into a different locus of the genome of $Y$. lipolytica thereby producing a new strain having an $U R A 3^{+}$phenotype.
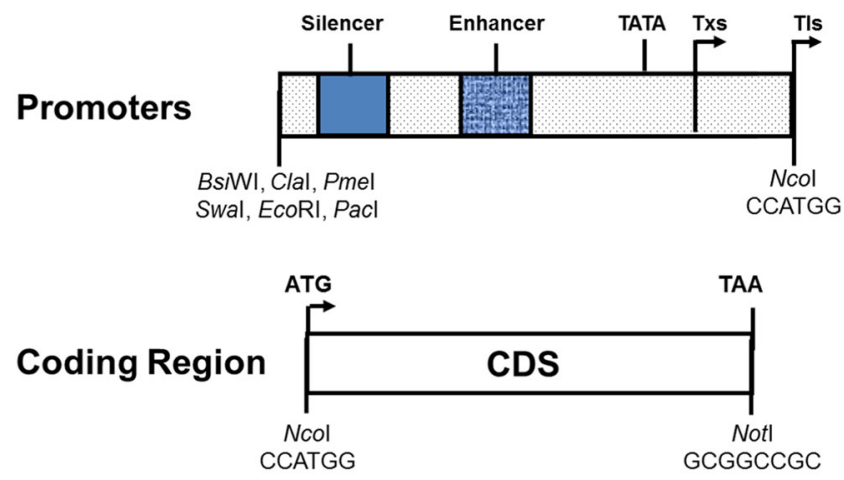

Terminator

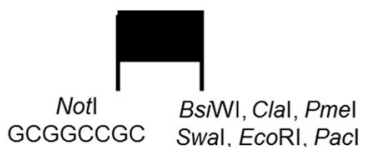

Fig. 3 Modular genetic elements for chimeric gene construction

Subsequent integration by homologous recombination with mutated ura 3 would produce a new $\mathrm{ura}^{-}$strain, identified with FOA-resistant selection. Thus, the URA3 gene (in combination with FOA selection) can be used as a selection marker in many rounds of transformation to introduce a large number of genes into the genome.

By increasing the copy number of the $\Delta 6$ pathway genes and "pushing" the carbon flux into the engineered pathway by overexpression of the $\mathrm{C}_{16 / 18}$-elongase gene of $M$. alpina (Macool et al. 2008) to convert the palmitic acid (C16:0) into stearic acid (C18:0) and the $\Delta 12$-desaturase gene of Fusarium moniliforme (Yadav et al. 2009) to convert the oleic acid to LA, we generated strain Y 9027 to produce EPA at about $40 \%$ of FAME (Zhu et al. 2010). This strain contained 19 copies of ten different heterologous genes that integrated into its genome. The in vivo substrate conversion catalyzed by the $\Delta 6$, $\Delta 5$, and $\Delta 17$ desaturases was about 86,90 , and $97 \%$, respectively, indicating that these enzymes functioned well in strain Y2097. The second highest fatty acid in the lipids was GLA, at about $21 \%$ of FAMEs. The substrate conversion from GLA to DGLA catalyzed by $\mathrm{C}_{18 / 20}$ elongase was only about $69 \%$ in that strain. The GLA is the product of $\Delta 6$ desaturase and the substrate of $\mathrm{C}_{18 / 20}$ elongase. The $\Delta 6$ desaturase introduces a double bond into the LA acyl chain esterified to a phospholipid backbone; the $\mathrm{C}_{18 / 20}$ elongase catalyzes the condensation of a malonyl group to GLA acyl chain esterified to coenzyme A (Domergue et al. 2003; Meesapyodsuk and Qiu 2012). There were four copies of $\mathrm{C}_{18 / 20}$-elongase genes driven by strong promoters in strain $\mathrm{Y} 2097$, suggesting that the $\mathrm{C}_{18 / 20^{-}}$ elongation reaction was a bottleneck in the engineered pathway. Thus, to reduce the GLA, amount in the lipids requires not only an efficient $\mathrm{C}_{18 / 20}$-elongase activity but also an active acyl exchange between the phospholipid and CoA pools in the ER membrane. To enhance the acyl-exchange process, we separately amplified genes encoding for acyl-CoA synthase (ACS), choline phosphotransferase (CPT1), diacylglycerol 
Fig. 4 Strategy for integration of multiple copies of genes into the chromosomes of $Y$. lipolytica. FOA 5-fluoroorotic acid, $M M$ minimal medium

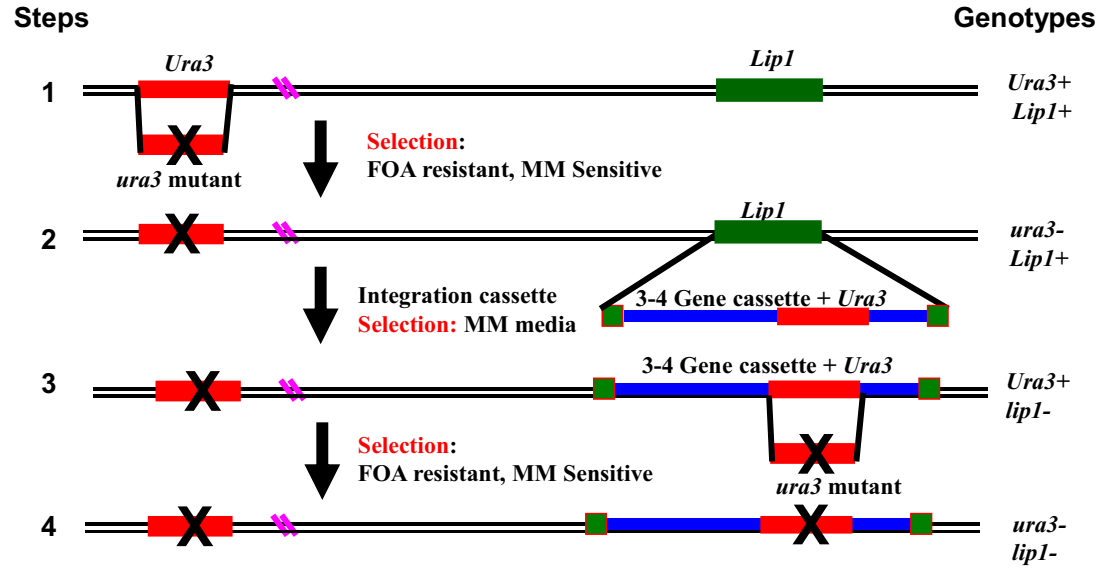

acyltransferase (DGAT1 and DGAT2), glycerol-3-phosphate acyltransferase (GPAT), lysophosphatidic acid acyltransferase (LPAAT), lysophosphatidylcholine acyltransferase (LPCAT), phospholipase C (PCL1), and phospholipase D (SPO22). These modifications each improved the GLA conversion efficiency up to $15 \%$. It is hypothesized that a combination of some of these genes could improve the efficiency of the $\mathrm{C}_{18 / 20}$ elongase, but it would not be able to eliminate the GLA, in engineered $Y$. lipolytica strains.

\section{Generation of EPA commercial production strains using the $\Delta 9$ pathway}

In order to reduce the level of omega- 6 fatty acids, especially the GLA, in lipids enriched with EPA, we decided to use the $\Delta 9$ pathway (Fig. 2). The selection of the $\Delta 9$ pathway ensured that the rate-limiting elongation is the first step of the engineered pathway; therefore, the accumulation of other intermediates should be kept to a minimum. The genes encoding $\Delta 9$ elongases (Damude et al. 2007) and $\Delta 8$ desaturases (Damude and Zhu 2007) were isolated and characterized from Euglena gracilis, Euglena anabaena, and Eutreptiella, sp. CCMP389. To increase the $\Delta 8$-desaturase activity, we also constructed three $\Delta 9$-elongase and $\Delta 8$ desaturase bifunctional fusion genes (Damude et al. 2008); the $\Delta 8$-desaturase activity in these fusion enzymes increased almost $100 \%$ compared with $\Delta 8$-desaturase alone while keeping similar $\Delta 9$-elongase activity. At the same time, three genes encoding $\Delta 5$ desaturases from $E$. gracilis, E. anabaena, and Eutreptiella, sp. CCMP626 (Pollak et al. 2012), and three genes encoding $\Delta 17$ desaturases from Pythium aphanidermatum, Phytophthora ramorum, and Phytophthora sojae were also isolated and studied for their activities and substrate selectivity (Xue et al. 2013b). Additionally, several genes encoding different acyltransferases (Zhang et al. 2012) were also isolated and used to improve fatty acid traffic in the ER.
To construct a high EPA production strain using the $\Delta 9$ pathway, we used a series of strategies. First, an efficient EPA biosynthetic pathway was built by using strong promoters such as EXP1, FBAINm, GPAT, GPD, and YAT, all heterologous genes were codon-optimized and multiple copies of structural genes were inserted for each step. To increase the $\Delta 8$-desaturase activity, several copies of $\Delta 9$-elongase and $\Delta 8$ desaturase bifunctional fusion genes were employed (Damude et al. 2008). Second, "pushing" and "pulling" the carbon flux into the engineered $\Delta-6$ pathway were achieved by overexpression of the $\mathrm{C}_{16 / 18}$-elongase gene (Macool et al. 2008) and the $\Delta 12$-desaturase gene (Yadav et al. 2009) and by using multiple copies of $\Delta 17$-desaturase genes (Xue et al. 2013b). Third, beta-oxidation was reduced by knockout of genes encoding for peroxins (Xue et al. 2013a; Hong et al. 2009) such as PEX3 or PEX10 that are involved in peroxisome biogenesis and matrix protein import. Fourth, fatty acid transport was controlled by fine regulation of different acyltransferases to increase fatty acid flux for EPA production and lipid accumulation.

Since Y. lipolytica strain ATCC \#20362 prefers nonhomologous end-joining over homologous recombination (Weterings and Chen 2008), we designed to screen 96 transformants for each parent strain/construct combination by gas chromatography (GC) analysis (Cahoon et al. 2001). The nonhomologous recombination arising from each transformation generated a library of transformants with diverse performance. Several beneficial traits for EPA and lipid production were found through screening this diversity. For example, knockout of the PEX10 gene (Xue et al. 2013a; Hong et al. 2009) was discovered to increase EPA titer in lipid to more than twice as those of its PEX+ siblings. We also discovered that deletion of the PEX10 gene in DGLA and ARA production strains could also more than double DGLA and ARA titers in lipids compared to the parent strains with a wild-type PEX10 gene (Xue et al. 2013a). In these strains with pex $10 \Delta$, beta-oxidation is greatly reduced and there were no normal peroxisomes inside cells. Unidentified membrane-like 
structures are observed that might be the deformed nonfunctional peroxisomes.

By combining the above strategies, we first generated the Gen I strain Y4305 (Xue et al. 2013a) that contains 30 copies of 9 different genes and produces EPA at $56.6 \%$ FAME, without GLA accumulation. The total EPA produced was about $15 \%$ of DCW. The lipid produced by strain Y4305 has a unique and healthy fatty acid profile that contains less than $5 \%$ saturated fatty acids and has only small amounts of intermediates. As is the case in strain Y2097 using the $\Delta 6$ pathway, the substrate conversion efficiency of the introduced desaturases is still significantly higher than the elongases in strain Y4305. However, the selection of the $\Delta 9$ pathway ensured that the rate-limiting elongation is the first step of the engineered pathway. Accumulation of intermediates is therefore minimized, in contrast to cells engineered with the $\Delta 6$ pathway where the first step is not rate limiting and accumulation of GLA becomes significant.

To increase the rate, titer, and yield for EPA production, we generated Gen II strain Z1978 (Hong et al. 2011a) via 24 steps of genetic modifications. It contained 35 copies of 17 different genes and produces EPA at $>58 \%$ FAME. The total EPA content in strain Z1978 is about $20 \%$ DCW. The fatty acid profile of strain Z1978 is similar to that of strain Y4305, with extremely low saturated fatty acids and only small amounts of intermediates and no GLA. Strain Z1978 has increased lipid contents over strain Y4305 from about $30 \%$ DCW in Y4305 strain to more than $38 \%$ DCW in strain Z1978.

Based on strain Z1978, we generated strain Gen III HP Z5567 (Hong et al. 2011a) by six more steps of metabolic engineering. Strain Z5567 contained 41 copies of 19 different genes. It produced EPA at about $50 \%$ FAME but with a total lipid of more than $50 \%$ DCW; therefore, strain Z5567 produced EPA at about $25 \%$ DCW, which is about 25 and $67 \%$ improvement over strain Z1978 and Y4305, respectively.

\section{Selection of production strains under fermentation conditions}

Fermentation process development as well as strain engineering plays a critically important role in converting the fundamental research to real commercial application. The fermentation research was initiated at the start of the strain engineering research. Fermentation experimentation included (1) strain screening under fermentation conditions, (2) optimization of fermentation conditions for each promising new strain, and (3) process scale-up. Figure 5 summarizes the typical workflow for the omega-3 fermentation research work. Figure 5a represents the generation of candidate strains by metabolic engineering in Yarrowia cells. Thousands of new or promising strains that are generated by metabolic engineering were tested sequentially in small-scale simple bioreactors (working volume $=1 \sim 100 \mathrm{~mL}$ ), which included 24-well blocks, test tubes, shake flasks, and micro-fermentors (Fig. 5b). The omega-3 fermentation is a two-stage (growth+lipid production/oleaginous) process. Therefore, cells are first grown in these simple bioreactors to a specified density after which they are deprived of nitrogen for growth and given glucose for maintenance and lipid production. At the end of the production stage, the lipid composition in the Yarrowia biomass, including EPA and other fatty acids, is determined by GC
Fig. 5 Workflow of omega-3 fermentation for both strain evaluation and process development. New strains generated by metabolic engineering (a) are sequentially evaluated in 24-well blocks, shake flasks, and microfermentors (b) before they are tested in lab-scale fermenters (c). Strains that perform well in labscale fermentors are further tested in pilot scale (d) before being adopted for EPA production in commercial-scale fermenters

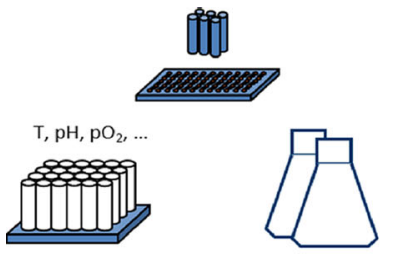

(b) 24-well plates, test tubes flasks, and micro-fermentors

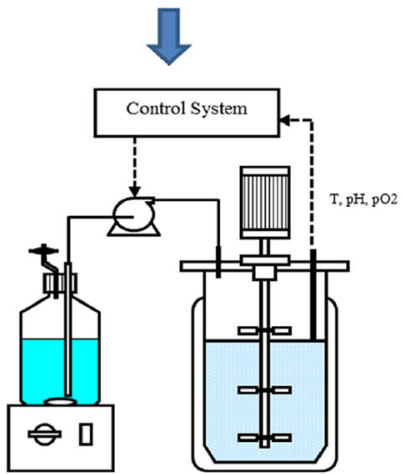

(c) Lab-Scale Fermentation

\section{Strain Evaluation}
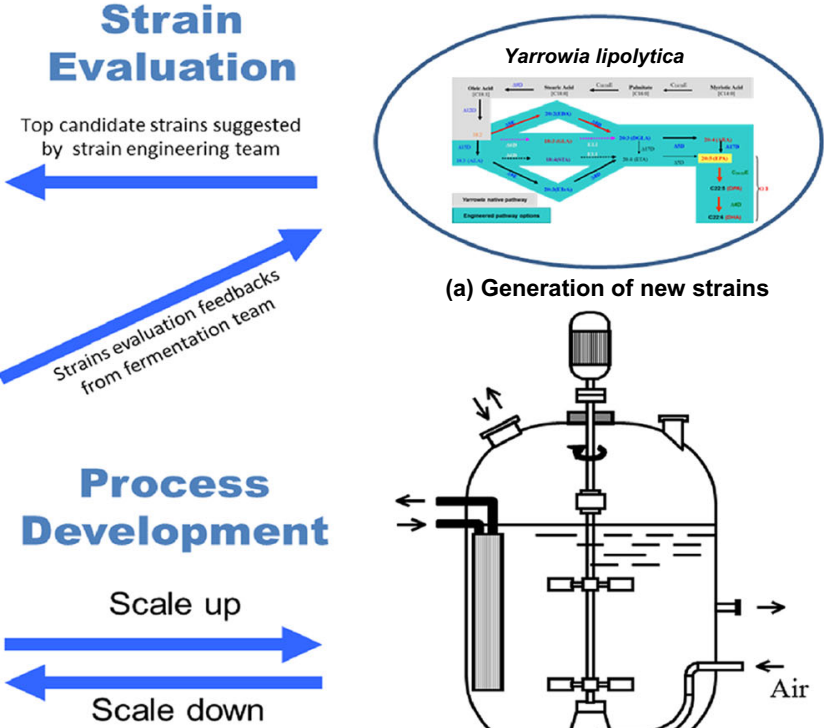

(a) Generation of new strains

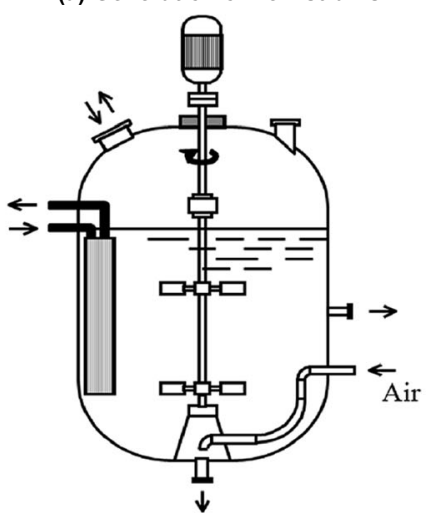

(d) Large Scale Fermentation 
analysis (Cahoon et al. 2001). In shake flask and microbioreactor experiments, dry cell weight (DCW) is also determined due to the availability of more sample volume $(\sim 5 \mathrm{~mL})$, so that the total lipid content of each sample (total lipid/DCW) is determined to better evaluate the biomass, lipid, and EPA productivities.

Dozens to hundreds of candidate strains that show high EPA content in lipid and high lipid content in biomass in the simple bioreactors are taken one step further to lab-scale fermenters $(2 \sim 10 \mathrm{~L})$, in which the dissolved oxygen, $\mathrm{pH}$ value, and especially substrate feed can be controlled more consistently than is possible in the simple bioreactors. The labscale fermentation results, including the time course data for EPA titer, rate, and yield, are used to determine the very top strains to be scaled up in pilot-scale fermentors under commercially achievable medium and process conditions (Fig. 5d).

The rate-limiting step in the workflow shown in Fig. 5 is the use of some simple bioreactors (24-well blocks, test tubes, flasks, and micro-fermentors) to identify the top strains or to prescreen medium and process conditions (Xie 2012). It is desirable to have a multi-bioreactor system with small working volumes to allow testing of thousands of candidate strains, yet each small bioreactor system must have high-quality process and feed controls so that the data obtained from these small bioreactors predict the performance in lab- and pilotscale fermentors.

The 24-well block or test tube is the simplest bioreactor, but its data is much less reliable due to the low controllability in a small volume and insufficient sample volume available for both lipid content and lipid composition analysis (Danielson et al. 2004; Stockmann et al. 2003). Shake flasks are also simple and easy to run at larger working volumes (10 $100 \mathrm{~mL}$ ) typically without monitoring and controlling $\mathrm{pH}$ values and dissolved oxygen (DO) levels, but they are relatively labor intensive to prepare and require fairly bulky shakers (Büchs 2001). Micro-titer plates/bioreactors have a large number of small reaction wells ( $1 \mathrm{~mL}$ or less) with each well's $\mathrm{pH}$ value and DO level possible to monitor, which is very efficient for high-throughput growth evaluation (Duetz et al. 2000; Amanullah et al. 2010). However, the precise controls of $\mathrm{pH}$ values and DO levels are still not available for most micro-titer/bioreactors on the market. The small working volume also limits the micro-titer bioreactor's application in the omega-3 project due to the insufficient biomass samples (less than $5 \mathrm{~mL}$ ) available for DCW and total lipid content analysis.

To minimize the limitations in control and to combine the high-throughput advantages of the 24-well blocks, test tubes, flasks, and micro-titer/bioreactors, we used an advanced micro-fermenter system for EPA strain screening and fermentation optimization (Xie 2012). A micro-fermenter is a highly integrated multi-fermentor system with a few milliliters of working volume per reactor; each reactor was independently controlled at the preset temperature, $\mathrm{pH}$ value, and $\mathrm{DO}$ level. There have been several different types of microfermenters reported in fermentation research work (Doig et al. 2005; Betts et al. 2006; Gilla et al. 2008). We used a Micro-24 bioreactor system for the EPA strain screening (Xie 2012). In this case, 24 individual micro-reactors, each with its own strain and process condition (T, pH, DO), can be processed at the same time. The high controllability of this system provided high-quality fermentation data for the end-of-run samples, including the by-product analysis, DCW, lipid content, EPA content in lipid, and EPA conversion yield. These data significantly increased the predictability of each individual strain's performance in lab- or large-scale fermentation, as shown in an example in Fig. 6.

\section{Optimization of lab-scale fermentation}

After we selected the top candidate strains by microfermentation analysis, we needed to further optimize the medium and process conditions (e.g., T, $\mathrm{pH}, \mathrm{DO}$, substrate feed) for a given strain to maximize its EPA production and minimize by-product formation (e.g., organic acids), in order to reduce the cost of manufacture (COM) under commercially achievable conditions. While flasks and micro-fermenters gave some guidance around optimization of the fermentation conditions, they are not sufficient for understanding, exploring, and further improving a complete fermentation run's titer, rate, yield, and cost of a fermentation process at large scale. For that purpose, the optimization work for a selected production strain was mainly conducted in lab-scale fermenters. Since the EPA lipid is an intracellular product of the Yarrowia biomass, the goal of optimization is first to maximize the biomass production in growth phase, and then to maximize EPA production and minimize by-product

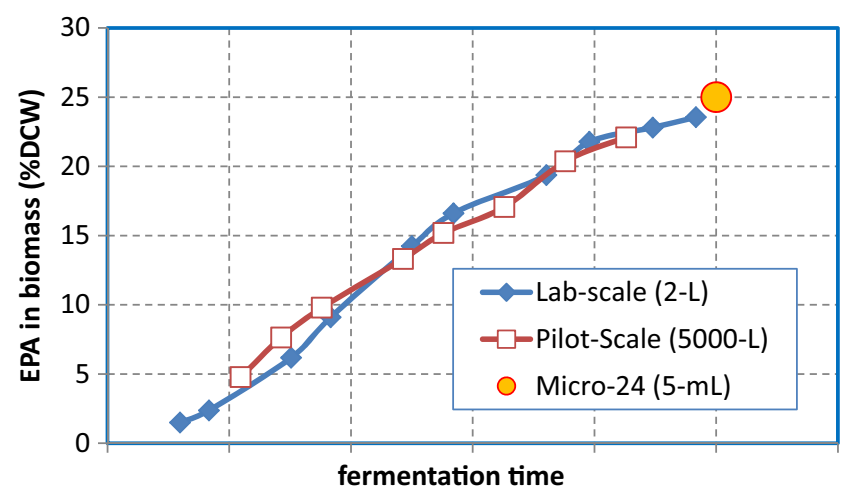

Fig. 6 EPA titer comparison of Gen III HP strain in typical runs of micro24 bioreactor $(5 \mathrm{~mL})$, lab-scale fermentor $(2 \mathrm{~L})$, and pilot-scale fermentor $(5,000 \mathrm{~L})$. The micro-fermentor achieved very consistent performance compared with the larger-scale fermentation 
formation in the oleaginous phase. We developed a two-stage (or two-phase) fed-batch fermentation process to maximize both biomass and EPA production, as shown in Fig. 7. In the first stage of the fermentation, the Yarrowia cells are grown on the carbohydrate substrate with nitrogen provided by both the organic nitrogen source (e.g., yeast extract) in the nutrient medium and the inorganic $\mathrm{NH}_{4} \mathrm{OH}$ used for $\mathrm{pH}$ control. After a certain period of time, the base for $\mathrm{pH}$ control is switched from $\mathrm{NH}_{4} \mathrm{OH}$ to $\mathrm{KOH}$ to restrict further nitrogen supply. Cell growth then stops after consuming the residual nitrogen in the medium, and the Yarrowia cells start to accumulate lipids by using the carbohydrate supplied. Our experience showed that the optimal conditions often vary when the strain is engineered in significantly different genetic backgrounds. Therefore, more optimization work is always expected to improve a newly selected strain's performance.

\section{Using modeling to guide fermentation process optimization and scale-up}

Though the optimization of lab-scale fermentation is critical for a selected production strain to achieve good performance at large scale, it is very time-consuming and labor-intensive. As we had accumulated more fermentation data and gained more understanding for both the strain and process, we used a set of mathematical equations, i.e., the dynamic models, to describe the fermentation behavior. A unique and also critical aspect of process development and scale-up for the EPA project was the use of dynamic models. Unstructured mathematical models were built from first principles, which included the model equations of cell growth, substrate consumption, nitrogen utilization, oxygen uptake, lipid and EPA formation, and by-product accumulation. The models were matched to the historical experimental data from many lab-scale and pilotscale fermentation experiments under different conditions. The models could predict cell growth, DCW, DO level, oxygen uptake rate (OUR), $\mathrm{CO}_{2}$ evolution rate (CER), and variables that were also measured during the fermentation as a function of various medium and process conditions. When a new production strain was applied, a few model parameters may need to be adjusted to keep the model's predictability based on the new experimental data and the understanding of the new strain. The dynamic models were thus able to predict the key performance parameters (e.g., titer, rate, and yield of a product) before and during the run and further help guide the fermentation optimization and process scale-up. Examples of the dynamic model's capability are shown in Fig. 8.

\section{Fermentation scale-up}

The last step in fermentation process development is the scaleup to pilot and then commercial scales. There are a few criteria commonly used for fermentation scale-up, including geometry similarity, power input, and mass transfer coefficient $K_{L a}$ (Shuler and Kargi 2002). For the EPA fermentation process, we often tested our selected production strain in the pilot-scale
Fig. 7 EPA production by a twostage fermentation developed primarily in lab-scale fermenters. In growth phase, nitrogen is mainly provided by $\mathrm{NH}_{4} \mathrm{OH}$ for $\mathrm{pH}$ control to build up biomass. During the production phase, nitrogen is limited by switching the ammonium base to $\mathrm{KOH}$ to produce lipid with high EPA content
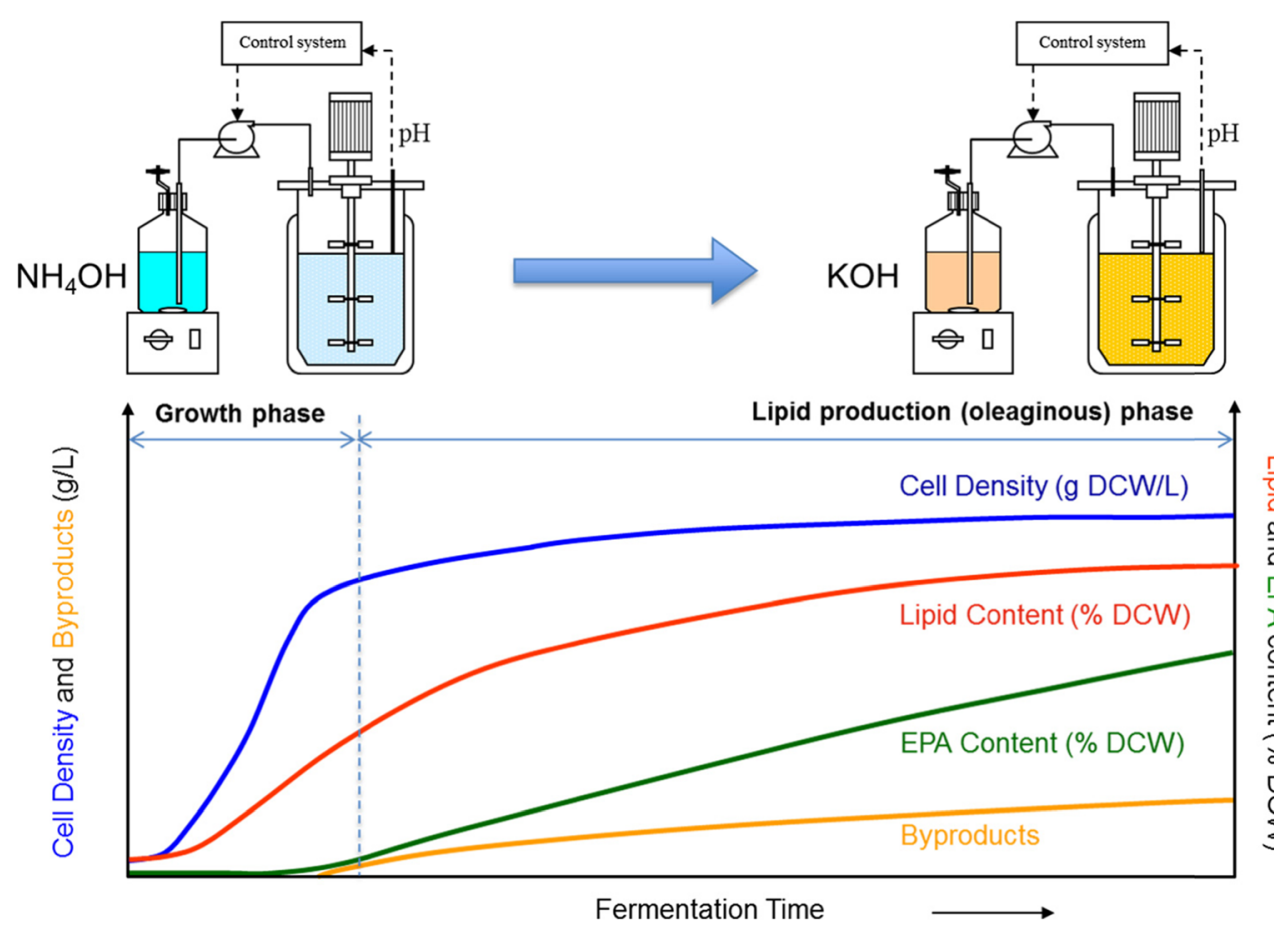
a

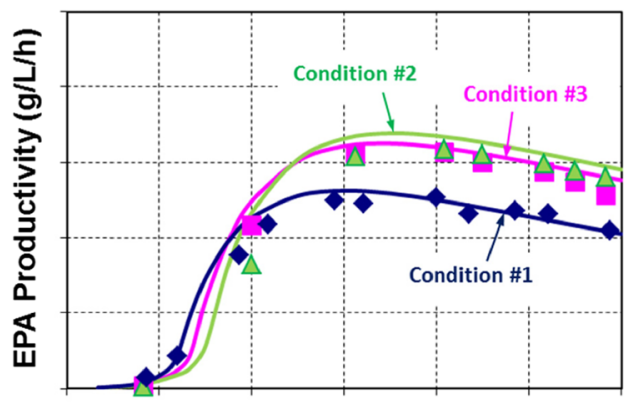

fermentation time

Fig. 8 Examples of using the predictive fermentation model for process optimization (a) and scale-up (b). a The model simulation (solid lines) guided the experiments (symbols) to significantly improve EPA productivities by changing the process condition from $\# 1$ to $\# 2$ and \#3.

facilities before we set a criterion for scale up, by which we were able to gather information of the pilot-scale fermentation's dynamic behavior. Besides the regular online data of temperature, $\mathrm{pH}$ value, feed rate, and DO levels, the dynamic information also included the agitation, aeration rate, and the mass transfer characteristics. Now, the benefits of highly predictive dynamic models became even more evident as we moved from the pilot plant to commercial production. We incorporated the agitation and aeration rate of the pilot-scale fermentation into the dynamic models so that we could study mass transfer characteristics as functions of superficial gas velocity and agitation power for each run. We then used the dynamic models to predict the commercial-scale fermentation's performance with the specific agitation and aeration in commercial-scale fermentors and thus guided the successful scale-up for the commercial production.

However, we quite often faced some challenges when there were some restrictions in the commercial scale fermentors, either for the use of some important medium components or for the process control that we could achieve at commercial scale. For example, the commercial scale fermentor uses only commercially available raw materials, which may have some other minor components affecting fermentation performance. Also, the much larger size of a commercial-scale fermentor causes significantly different fluid dynamic behavior in the reactor. To understand how a difference of a commercial-scale fermentor affects the fermentation results, we designed a series of lab-scale experiments to mimic the fermentation with the medium and/or process conditions at commercial scale. These are called "scale-down" studies (Ozbek 1997; Nienow et al. 2011). By the scale-down studies, we were able to identify a few important factors that affecting the scale-up. Then, we either sent the feedback to the strain-engineering team to reengineer the strain or modified our fermentation protocols for the lab-scale and pilot-scale experiments to fix the possible scale-up problems, as previously indicated in Fig. 5. b

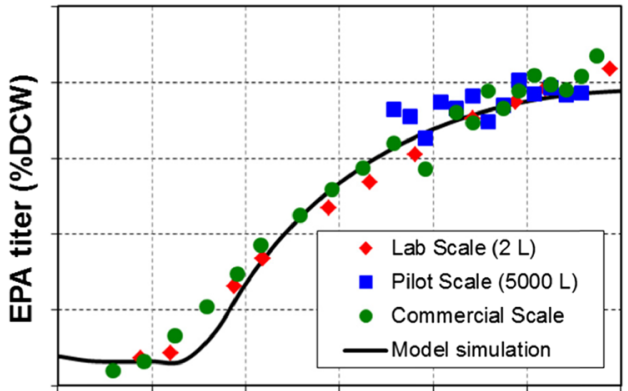

fermentation time

b The model (solid line) was used to design the scale-up fermentation conditions so that very comparable results (symbols) were achieved in lab-, pilot-, and commercial-scale fermentors

\section{Conclusions}

The Y. lipolytica yeast was engineered in DuPont to produce a high level of EPA in biomass under commercial-scale fermentation conditions. By overexpressing a combination of enzymes that are necessary for synthesis of EPA via the $\Delta 9 / \Delta 8$ pathway and for optimization of lipid metabolism, the Gen III HP strain was created that is capable of making EPA at $25 \%$ DCW and at more than $50 \%$ lipids. The high level of EPA production was achieved through careful balancing of the expression levels of various pathway enzymes, and modification of fatty acid and lipid metabolism of the host. Disruption of the peroxisome biogenesis gene had a major positive impact on the production of EPA and the metabolism of storage lipid, as well as reduction of the major by-products.

Both research studies in strain engineering and fermentation process development were initiated at the same time to help convert the lab research results to commercial production. Advanced micro-fermentors with well-controlled process parameters significantly improved the efficiency of strain screening and the predictability of the selected strains' fermentation performance at much larger scale. A two-stage fed-batch fermentation process was developed to maximize both biomass and EPA production and minimize the byproduct formation. Finally, mathematical modeling of the developed fermentation process and scale-down studies played critically important roles in the successful process scale-up. Two commercial products, New Harvest $^{\mathrm{TM}}$ EPA oil and Verlasso ${ }^{\circledR}$ salmon, were developed using our sustainable EPA source. Our journey demonstrated the power of modern biotechnology by combining both fundamental scientific research and industrial engineering.

Acknowledgments We are grateful to Dr. Henry Bryndza and Dr. Michael Saltzberg for their strong support. We thank all members in the 
omega-3 technical, manufacture, and business teams for their contributions and discussions.

Open Access This article is distributed under the terms of the Creative Commons Attribution License which permits any use, distribution, and reproduction in any medium, provided the original author(s) and the source are credited.

\section{References}

Abghari A, Chen S (2014) Yarrowia lipolytica as an oleaginous cell factory platform for production of fatty acid-based biofuel and bioproducts. Front. Front Energy Res 2:1-21. doi:10.3389/fenrg. 2014.00021

Alper H, Fischer C, Nevoigt E, Stephanopoulos G (2005) Turning genetic control through promoter engineering. Proc Natl Acad Sci U S A 102:12678-12683

Amanullah A, Otero JM, Mikola M, Hsu A, Zhang J, Aunins J, Schreyer HB, Hope JA, Russo AP (2010) Novel micro-bioreactor high throughput technology for cell culture process development: reproducibility and scalability assessment of fed-batch $\mathrm{CHO}$ cultures. Biotechnol Bioeng 106:57-67

Ballantyne CM, Bays HE, Kastelein JJ, Stein E, Isaacsohn JL, Braeckman RA, Soni PN (2012) Efficacy and safety of eicosapentaenoic acid ethyl ester (AMR101) Therapy in StatinTreated Patients With Persistent High Triglycerides (from the ANCHOR Study). Am J Cardiol 110:984-992

Barth G, Gaillardin C (1996) Yarrowia lipolytica. In: Wolf K (ed) Nonconventional yeasts in biotechnology. Springer, Berlin, Heidelberg, New York, pp 313-388

Barth G, Gaillardin C (1997) Physiology and genetics of the dimorphic fungus Yarrowia lipolytica. FEMS Microbiol Rev 19:219-237

Beopoulos A, Cescut J, Haddouche R, Uribelarrea JL, Molina-Jouve C, Nicaud JM (2009) Yarrowia lipolytica as a model for bio-oil production. Prog Lipid Res 48:375-387

Beopoulos A, Desfougeres T, Sabirova J, Nicaud JM (2010) Yarrowia lipolytica as a cell factory for oleochemical biotechnology. In: Timmis KN (ed) Handbook of Hydrocarbon and Lipid Microbiology. Springer, Berlin

Betts J, Doig SD, Baganz F (2006) The characterization and application of a miniature $10 \mathrm{ml}$ stirred-tank bioreactor, showing scale-down equivalence with a conventional $7 \mathrm{~L}$ reactor. Biotechnol Prog 22: 681-688

Blank LM, Lehmbeck F, Sauer U (2005) Metabolic-flux and network analysis in fourteen hemiascomycetous yeasts. FEMS Yeast Res 5: $545-558$

Büchs J (2001) Introduction to advantages and problems of shaken cultures. Biochem Eng J 7:91-98

Cahoon EB, Ripp KG, Hall SE, Kinney AL (2001) Formation of conjugated $\Delta 8, \Delta 10$-double bonds by $\Delta 12$-oleic acid desaturase-related enzymes: biosynthetic origin of calendic acid. J Biol Chem 276: 2637-2643

Calder PC (2006) n-3 Polyunsaturated fatty acids, inflammation, and inflammatory diseases. Am J Clin Nutr 83(suppl):1505S-1519S

Chacon-Lee TL and Gonzalez-Marino GE (2010) Microalgae for "Healthy" Foods-Possibilities and Challenges. Comprehensive Reviews in Food Science and Food Safety 9: 655-675. doi:10. 1111/j.15414337.2010.00132.x

Chen DC, Beckerich JM, Gaillardin C (1997) One-step transformation of the dimorphic yeast Yarrowia lipolytica. Appl Microbiol Biotechnol 48:232-235

Damude HG, Zhu Q (2007) Delta-8 desaturase and its use in making polyunsaturated fatty acids. US Patent US 7256033
Damude HG, McGonigle B, Zhu Q, Zhixiong X (2007) Delta-9 elongases and their use in making polyunsaturated fatty acids. US Patent Application US20070118929

Damude HG, Kinney AJ, Ripp KG, Zhu QQ (2008) Multizymes and their use in making polyunsaturated fatty acids. US Patent Application 20080254191

Danielson PB, Büchs J, Stockmann C, Fogleman JC (2004) Maximizing cell densities in miniprep-scale cultures with $\mathrm{H} 15$ medium and improved oxygen transfer. Biochem Eng J 17:175-180

Davidow LS, O'Donnell MM, Kaczmarek FS, Pereira DA, DeZeeuw JR, Franke AE (1987a) Cloning and sequencing of the alkaline extracellular protease gene of Yarrowia lipolytica. J Bacteriol 169:46214629

Davidow LS, Kaczmarek FS, DeZeeuw JR, Conlon SW, Lauth MR, Pereira DA, Franke AE (1987b) The Yarrowia lipolytica LEU2 gene. Curr Genet 11:377-383

Doig SD, Diep A, Baganz F (2005) Characterisation of a novel miniaturized bubble column bioreactor for high throughput cell cultivation. Biochem Eng J 23:97-105

Domergue F, Abbadi A, Ott C, Zank TK, Zahringer U, Heinz E (2003) Acyl carriers used as substrates by the desaturases and elongases involved in very long-chain polyunsaturated fatty acids biosynthesis reconstituted in yeast. J Biol Chem 278:35115-35126

Duetz WA, Ruedi L, Hermann R, O'Connor K, Büchs J, Witholt B (2000) Methods for intense aeration, growth, storage and replication of bacterial strains in microtiter plates. Appl Environ Microbiol 66: 2641-2646

Dujon B, Sherman D, Fischer G, Durrens P, Casaregola S, Lafontaine I, Montigny J, Marck C, Neuvéglise C, Talla E, Goffard N, Frangeul L, Aigle M, Anthouard V, Babour A, Barbe V, Barnay S, Blanchin S, Beckerich JM, Beyne E, Bleykasten C, Boisramé A, Boyer J, Cattolico L, Confanioleri F, Daruvar A, Despons L, Fabre E, Fairhead C, Ferry-Dumazet H, Groppi A, Hantraye F, Hennequin C, Jauniaux N, Joyet P, Kachouri R, Kerrest A, Koszul R, Lemaire M, Lesur I, Ma L, Muller H, Nicaud JM, Nikolsk M, Oztas S, OzierKalogeropoulos O, Pellenz S, Potier S, Richard GF, Straub ML, Suleau A, Swennen D, Tekaia F, Wésolowski-Louvel M, Westhof E, Wirth B, Zeniou-Meyer M, Zivanovic I, Bolotin-Fukuhara M, Thierry A, Bouchier C, Caudron B, Scarpelli C, Gaillardin C, Weissenbach J, Wincker P, Souciet JL (2004) Genome evolution in yeasts. Nature 430:35-44

Fournier P, Abbas A, Chasles M, Kudla B, Ogrydziak DM, Yaver D, Xuan J-W, Peito A, Ribet A-M, Feynerol C, He F, Gaillardin C (1993) Colocalization of centromeric and replicative functions on autonomously replicating sequences isolated from yeast Yarrowia lipolytica. Proc Natl Acad Sci U S A 90:4912-4916

Gilla NK, Appletonb M, Baganza F, Lye GJ (2008) Design and characterisation of a miniature stirred bioreactor system for parallel microbial fermentations. Biochem Eng J 39:164-176

Groenewald M, Boekhout T, Neuvéglise C, Gaillardin C, van Dijck PW, Wyss M (2014) Yarrowialipolytica: safety assessment of an oleaginous yeast with a great industrial potential. Crit Rev Microbiol 40: 187-206. doi:10.3109/1040841X

Hong SP, Sharpe PL, Xue Z, Yadav NS, Zhu QQ (2009) Peroxisome biogenesis factor protein (pex) disruptions for altering polyunsaturated fatty acids and total lipid content in oleaginous eukaryotic organisms. US Patent Application 20090117253

Hong S-Y, Seip J, Sharpe PL, Xue Z, Yadav NS, Zhang H, Zhu QQ (2011a) Recombinant microbial host cells for high eicosapentaenoic acid production. U.S. Patent Application 20120052537

Hong S-Y, Seip J, Walters-Pollak D, Rupert R, Jackson R, Xue Z, Zhu Q (2011b) Engineering Yarrowia lipolytica to express secretory invertase with strong FBA1IN promoter. Yeast 29:59-72

Jefferson RA, Kavanagh TA, Bevan MW (1987) GUS fusions: betaglucuronidase as a sensitive and versatile gene fusion marker in higher plants 
Juretzek T, Le Dall M, Mauersberger S, Gaillardin C, Barth G, Nicaud J (2001) Vectors for gene expression and amplification in the yeast Yarrowia lipolytica. Yeast 18:97-113

Kapoor R, Patil UK (2011) Importance and production of omega-3 fatty acids from natural sources. Int Food Res J 18:493-499

Knutzon DS, Thurmond JM, Huang YS, Chaudhary S, BobikEGJr CGM, Kirchner SJ, Mukerji P (1998) Identification of delta5-desaturase from Mortierella alpina by heterologous expression in bakers' yeast and canola. J Biol Chem 273:29360-29366

Kyle DJ (2001) The large-scale production and use of single-cell highly enriched oil in docosahexaenoic acid. In: Shahidi F, Finley J (Eds) Omega-3 fatty acids: chemistry, nutrition, and health effects. Oxford University Press, pp 92-107

Macool, DJ, Xue Z, Zhu Q (2008) A Mortierellaalpina C16/18 fatty acid elongase. US Patent 7470532

Martins DA, Custódio L, Barreira L, Pereira H, Ben-Hamadou R, Varela J, Abu-Salah KM (2013) Alternative sources of n-3 long-chain polyunsaturated fatty acids in marine microalgae. Mar Drugs 11: 2259-2281. doi:10.3390/md11072259

Mauersberger S, Wang HJ, Gaillardin C, Barth G, Nicaud JM (2001) Insertional mutagenesis in the $n$-alkane-assimilating yeast Yarrowia lipolytica: generation of tagged mutations in genes involved in hydrophobic substrate utilization. J Bacteriol 183:5102-5109

Meesapyodsuk D, Qiu X (2012) The front-end desaturase: structure, function, evolution and biotechnological use. Lipids 47:227-237

Metz JG, Roessler P, Facciotti D, Levering C, Dittrich F, Lassner M, Valentine R, Lardizabal K, Domergue F, Yamada A, Yazawa K, Knauf V, Browse J (2001) Production of polyunsaturated fatty acids by polyketide synthases in both prokaryotes and eukaryotes. Science 293:290-293

Muller S, Sandal T, Kamp-Hansen P, Dalboge H (1998) Comparison of expression systems in the yeasts Saccharomyces cerevisiae, Hansenula polymorpha, Klyveromyces lactis, Schizosaccharomyces pombe and Yarrowia lipolytica. Cloning of two novel promoters from Yarrowia lipolytica. Yeast 14:1267-1283

Nicaud JM (2012) Yarrowia lipolytica. Yeast 29:409-418

Nienow AW, Nordkvist M, Boulton CA (2011) Scale-down/scale-up studies leading to improved commercial beer fermentation. Biotechnol J 6:911-925

Ozbek B (1997) Mathematical modeling and simulation studies of scaledown fermentation systems. Chem Eng Technol 20:259-267

Pérez-Campo FM, Domínguez A (2001) Factors affecting the morphogenetic switch in Yarrowia lipolytica. Curr Microbiol 43:429-433

Picataggio SK, Zhu QQ (2007) Glyceraldehyde-3-phosphate dehydrogenase and phosphoglycerate mutase regulatory sequences for gene expression in oleaginous yeast. US Patent 7459546

Picataggio SK, Yadav NS, Zhu QQ (2007) Production of polyunsaturated fatty acids in oleaginous yeasts. US Patent 7238482

Pollak DW, Bostick MW, Yoon H, Wang J, Hollerbach DH, He H, Damude HG, Zhang H, Yadav NS, Hong SP, Sharpe P, Xue Z, \& Zhu Q. (2012) Isolation of a delta 5 Desaturase Gene from Euglena gracilis and Functional Dissection of Its HPGG and HDASH Motifs. 47:913-26

Ratledge C (2005) Single cell oils for the 21st century. In: Cohen Z, Ratledge C (eds) Single cell oils. AOCS Press, Champaign, Illinois, pp 1-20

Sayanova O, Napier JA (2004) Eicosapentaenoic acid: biosynthetic routes and the potential for synthesis in transgenic plants. Phytochemical 65:147-158
Schmid-Berger N, Schmid B, Barth G (1994) Ylt1, a highly repetitive retrotransposon in the genome of the dimorphic fungus Yarrowia lipolytica. J Bacteriol 176:2477-2482

Shuler ML, Kargi F (2002) Biorocess Engineering, 2nd edn. Prentice Hall PTR, Upper Saddle River, NJ, pp 286-306

Stockmann C, Losen M, Dahlems U, Knocke C, Gellissen G, Büchs J (2003) Effect of oxygen supply on passaging, stabilizing and screening of recombinant Hansenula polymorpha production strains in test tube cultures. FEMS Yeast Res 4:195-205

Szabo R, Stofaníková V (2002) Presence of organic sources of nitrogen is critical for filament formation and $\mathrm{pH}$-dependent morphogenesis in Yarrowia lipolytica. FEMS Microbiol Lett 206:45-50

Tai M, Stephanopoulos G (2013) Engineering the push and pull of lipid biosynthesis in oleaginous yeast Yarrowia lipolytica for biofuel production. Metab Eng 15:1-9

Wallis JG, Browse J (1999) The Delta8-desaturase of Euglena gracilis: an alternate pathway for synthesis of 20-carbon polyunsaturated fatty acids. Arch Biochem Biophys 365:307-316

Wen Z, Chen F (2005) Prospects for eicosapentaenoic acid production using microorganisms. In: Cohen Z, Ratledge C (eds) Single cell oils. AOCS Press, Champaign, Illinois, pp 138-160

Weterings E, Chen DJ (2008) The endless tale of non-homologous endjoining. Cell Res 18:114-124

Xie D (2012) Using an advanced microfermentor system for strain screening and fermentation optimization. In: Cheng Q (ed) Methods in molecular biology, 834 (Microbial Metabolic Engineering). Humana Press, New York, pp 217-231

Xuan J, Fournier P, Gaillardin C (1988) Cloning of the LYS5 gene encoding saccharopine dehydrogenase from the yeast Yarrowia lipolytica by target integration. Curr Genet 14:15-21

Xue Z, Zhu QQ (2012) Ammonium transporter promoter for gene expression in oleaginous yeast. US Patent 8323960

Xue Z, Sharpe PL, Hong SP, Yadav NS, Xie D, Short DR, Damude HG, Rupert RA, Seip JE, Wang J, Pollak DW, Bostick MW, Bosak MD, Macool DJ, Hollerbach DH, Zhang H, Arcilla DM, Bledsoe SA, Croker K, McCord EF, Tyreus BD, Jackson EN, Zhu Q (2013a) Production of omega-3 eicosapentaenoic acid by metabolic engineering of Yarrowia lipolytica. Nat biotech 31:734-740. doi:10. $1038 /$ nbt. 2622

Xue Z, He H, Hollerbach D, Macool DJ, Yadav NS, Zhang H, Szostek B, Zhu Q (2013b) Identification and characterization of new $\Delta-17$ fatty acid desaturases. Appl Microbiol Biotechnol 97:1973-1985. doi:10. 1007/s00253-012-4068-2

Yadav NS, Zhu Q, Zhang H (2009) $\Delta 12$ desaturases suitable for altering levels of polyunsaturated fatty acids in oleaginous yeast. US Patent 7504259

Yokoyama M, Origasa H, Matsuzaki M, Matsuzawa Y, Saito Y, Ishikawa Y, Oikawa S, Sasaki J, Hishida H, Itakura H, Kita T, Kitabatake A, Nakaya N, Sakata T, Shimada K, Shirato K (2007) Effects of eicosapentaenoic acid on major coronary events in hypercholesterolaemic patients (JELIS): a randomised open-label, blinded endpoint analysis. Lancet 369:1090-1098

Zhang H, Damude HG, Yadav NS (2012) Three diacylglycerol acyltransferases contribute to oil biosynthesis and normal growth in Yarrowia lipolytica. Yeast 29:25-38. doi:10.1002/yea.1914

Zhu Q, Xue Z, Yadav N, Damude H, Pollak D, Rupert R, Seip J, Hollerbach D, Macool D, Zhang H (2010) Metabolic engineering of an oleaginous yeast for the production of omega-3 fatty acids. In: Cohen Z, Ratledge C (eds) Single cell oils, 2nd edn. AOCS Press, Urbana Illinois, pp 51-73 